\title{
Integrated Control Based on Three-system of Vehicle Chassis
}

\author{
Lei Zhang ${ }^{\mathrm{a}}{ }^{*}$, Xiaoling $\mathrm{Jin}^{\mathrm{b}}$ and En-guo Dong ${ }^{\mathrm{c}}$ \\ Automotive School, Tianjin University of Technology and Education, 300222, Tianjin, China \\ azldeg@126.com, b1531543362@qq.com, cdegzl@sohu.com
}

Keywords: Chassis; integrated control; suspension; steering system; brake system

Abstract. The integrated control model based on two chassis subsystems has been widely studied and achieved a good vehicle dynamic performance; however these models haven't effectively improved the vehicle performance under some complex working conditions. In order to improve the overall vehicle performance, an integrated controller based on suspension system, steering system and brake system has been proposed. In the integrated controller the longitudinal, lateral and vertical dynamic characteristics of vehicle have been considered and the simulations for vehicle dynamic performance have been completed using Matlab/Simulink. The integrated controller includes eight input parameters, such as the yawing angular velocity, the dynamic tire load and the lateral vehicle acceleration, etc; and 6 output parameters including the control force for suspension, the additional yaw moment, etc. The simulation results show that the integrated controller effectively improves vehicle handling stability and driving safety under some complex working conditions.

\section{Introduction}

An integrated control model of two chassis subsystems has been widely studied and achieved a good vehicle dynamic performance. However, the kind of integrated control model has some problems. The generalized predictive control algorithm in the integrated model of suspension and steering system had made the actual vehicle movement tracks accurately track the ideal tracks, but the changes of vertical tire load might not be conducive to the ground adhesion required by the direct yaw-moment control (DYC) [1,2]; Moreover, the changes of roll characteristic in a high-speed turning also had disturbed the vehicle's turning track and handling stability $[3,4]$. The brake sideslip had been shortened when the brake was operated in a vehicle turning [5], however, the movement interference produced from the vehicle lateral, longitudinal and vertical force of chassis in a turning was inevitable. In order to resolve these problems, an integrated control model of automobile chassis with suspension system, braking system, and steering system has been built in which the coordinated control in the direction of longitudinal, lateral and vertical has been comprehensively considered.

\section{Strategy on the Controller Based on Three-system}

The integrated controller based on three-system for chassis is shown as Fig.1.

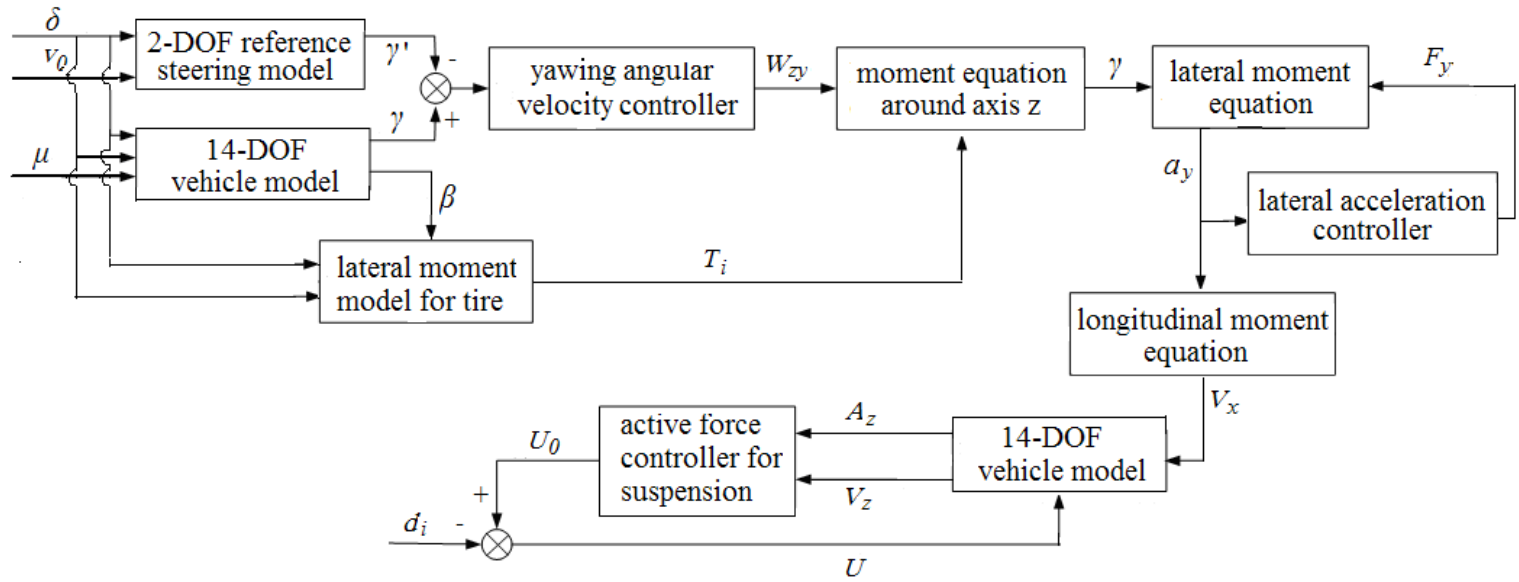

Fig.1 Integrated controller for chassis 
In Fig. $1, \delta$-the steering angle of steering wheel, $v_{0}$-initial velocity, $\mu$-road displacement, $\gamma$-the actual yawing angular velocity, $\gamma^{\prime}$-the ideal yawing angular velocity, $W_{z y}$-the additional yawing moment, $a_{y}$-lateral acceleration, $F_{y}$-lateral control force, $d_{i}$-dynamic wheel load, $A_{z}$-body acceleration, $V_{z}$-body velocity, $U$-the active force for suspensions, $V_{x}$-longitudinal velocity.

The integrated controller includes three independent controllers for suspension system, steering system and brake system. Compared to the integration model based on braking system and steering system, the three-system model improves the maximum longitudinal adhesion and tire cornering characteristics and it is more suitable to control brake safety in a turning because of the adding control for suspension system. Compared to the integrated model based on suspension and braking system, the three-system model decreases vehicle sideslip and improves driving safety because of the adding control for steering system.

\section{Controller Design}

Controller for Active Suspension. The changes caused by the vertical suspension displacement and the longitudinal acceleration directly result in the imbalance force for suspension [6], and produce the dynamic tire load $d_{1}, d_{2}, d_{3}, d_{4}$ which make vehicle instability. Therefore the controller chooses the body velocity $V_{z}$ and the body acceleration $A_{z}$ as the input parameters, and the output is the active force for suspensions $u_{f l}, u_{f r}, u_{r l}, u_{r r}$ as shown in Fig. 2 .

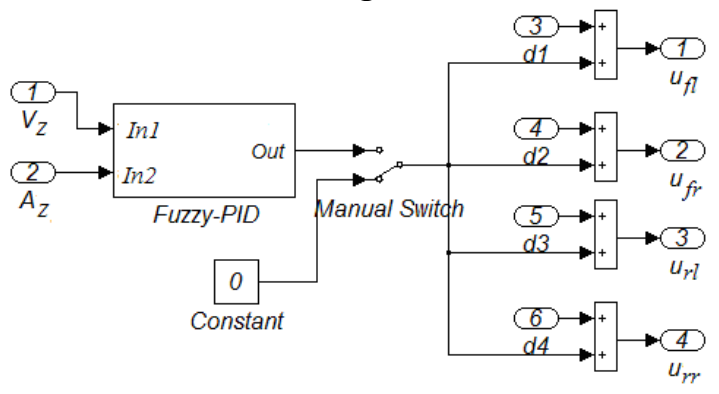

Fig.2 Controller for active suspension

In the suspension controller, Fuzzy PID control algorithm is used to control the dynamic tire load redistribution. The deviation is $e(t)=0-\ddot{Z}=-\ddot{Z}$ as the objective is close to 0 . In addition, the control rule is shown as Equ.1

$$
u(t)=K_{p} e(t)+K_{i} \int_{0}^{t} e(t) d t+K_{d} \frac{d e(t)}{d t}
$$

where, $K_{p}, K_{i}, K_{d}$-the proportional gain coefficient, the integral gain coefficient and the differential gain coefficient; $e(t)$-the difference value between feedback signal and input signal.

Lastly the setting parameters of PID are $K_{p}=240 ; K_{i}=135 ; K_{d}=0.01$.

Controller for Steering System. In the steering controller the additional yawing moment $w_{z y}$ is used as the controlled parameter in according with the difference value between the ideal yawing angular velocity $\gamma^{\prime}$ and the actual yawing angular velocity $\gamma$, as shown in Fig. 3 .

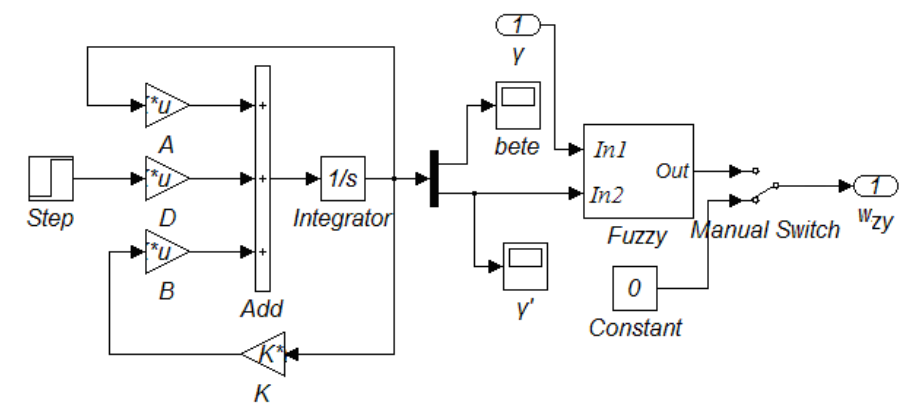

Fig.3 Controller for steering system 
In Fig.3, the ideal yawing angular velocity is the optimal value solved by the 2-DOF steering system, and the relevance vector is as the following

$$
\mathbf{A}=\left[\begin{array}{cc}
\frac{2\left(K_{\alpha f}+K_{\alpha r}\right)}{M V} & -1+\frac{2\left(L_{f} K_{\alpha f}-L_{r} K_{\alpha r}\right)}{M V^{2}} \\
\frac{2\left(L_{f} K_{\alpha f}-L_{r} K_{\alpha r}\right)}{I_{z}} & \frac{2\left(L_{f}{ }^{2} K_{\alpha f}+L_{r}{ }^{2} K_{\alpha r}\right)}{I_{z} V}
\end{array}\right] \quad \mathbf{B}=\left[\begin{array}{c}
-\frac{2 K_{\alpha r}}{M V} \\
\frac{2 L_{r} K_{\alpha r}}{I_{z}}
\end{array}\right] \quad \mathrm{D}=[0] .
$$

The basic range of input and output variables are respectively $e \in[-0.3,0.3], e c \in[-1.5,1.5]$, $u \in[-1800,1800]$. The domains of input $(E, E C)$ and output $(U)$ are defined as the followings: $\{3,2,1$, $0,1,2,3\}$. The input and output variables include five fuzzy subsets $\{\mathrm{NB}, \mathrm{NS}, \mathrm{O}, \mathrm{PS}, \mathrm{PB}\}$, NB-negative big, NS-negative small, O-zero, PS-positive small, PB-positive big. The membership functions of fuzzy variable $E, E C, U$ use the triangle membership function. The fuzzy control rules are shown in table 1.

Table1 Fuzzy control rules

\begin{tabular}{|c|c|c|c|c|c|}
\hline EC & \multirow{2}{*}{$\mathrm{NB}$} & NS & $\mathrm{O}$ & PS & PB \\
\hline NB & PB & PB & PB & PS & NB \\
\hline NS & PB & PS & PS & O & NB \\
\hline O & PB & PS & O & NS & NB \\
\hline PS & PB & O & NS & NS & NB \\
\hline PB & PB & NS & NB & NB & NB \\
\hline
\end{tabular}

The weighted matrix is respectively $Q=[150], R=[5 e 3]$, and the optimal feedback gain matrix is $K=[-0.0546,-0.0859]$.

Controller for Brake System. The braking system controller has considered the lateral force for vehicle as a braking in a vehicle turning. The vehicle's lateral acceleration $a_{y}$ is applied as the input parameters, and the lateral control force $F_{y}$ is used as the calculated data of PID, as shown in Fig.4. The setting parameters are $K_{p}=1550, K_{i}=256, K_{d}=0$.

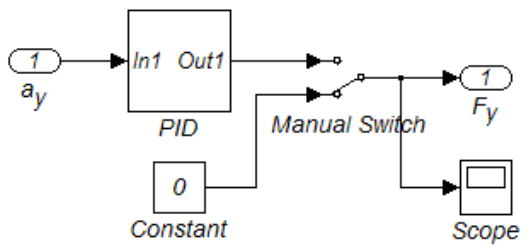

Fig.4 Controller for brake system

\section{Simulation Result Analyses}

The simulations have been completed using software of Matlab/Simulink. The initial simulation velocity of vehicle is $20 \mathrm{~m} / \mathrm{s}$. After simulation time reaches $1 \mathrm{~s}$, the step angle of $0.58 \mathrm{rad}$ for the steering wheel is as input. Until simulation time reaches $6 \mathrm{~s}$, the braking is operated.

To compare with the vehicle dynamic performance in the different models including passive, two-system and three-system, the body sideslip angle, the yawing angular velocity and the lateral acceleration have been analyzed as shown in Fig.5. In the figures, "I" represents the passive system, " $\Pi$ " represents the integration system based on the steering and active suspension and " $"$ " represents the integrated system based on the steering, active suspension and brake system.

In Fig.5 (a), the steady state value of the body sideslip angle respectively is about $-0.07 \mathrm{rad}$, $-0.025 \mathrm{rad}$ and-0.01 rad in the models of passive, two-system and three-system. The body sideslip angle in the three-system model is respectively decreased $85.7 \% 4$ and $37.5 \%$ than that in the passive model and the two-system model. The smaller body sideslip angle makes the body more quickly stable and improves the driving comfort and safety. 


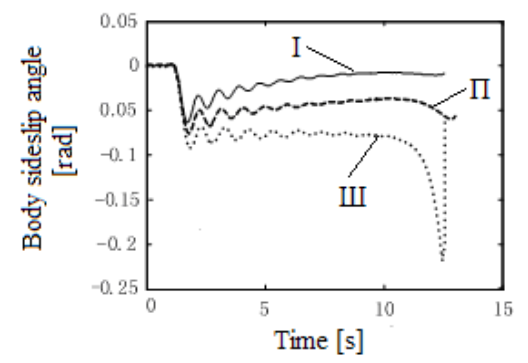

(a)Body sideslip angle

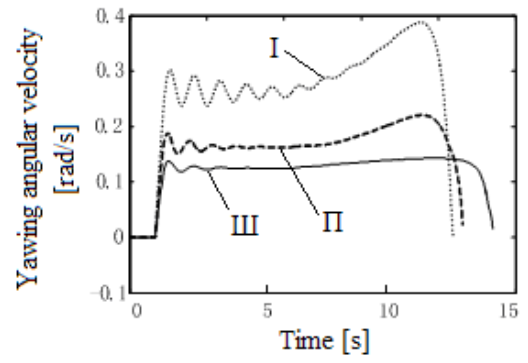

(b) Lateral acceleration

Fig. 5 Simulation results

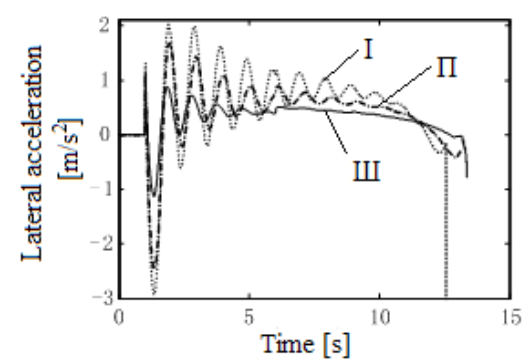

(c) Yawing angular velocity

In Fig.5 (b), the maximal peak value of the yawing angular velocity is respectively $0.38 \mathrm{rad} / \mathrm{s}$, $0.22 \mathrm{rad} / \mathrm{s}$ and $0.14 \mathrm{rad} / \mathrm{s}$ in the models of passive, two-system and three-system. The body stability has been improved because of the decreased yawing angular velocity.

In Fig.5 (c), the maximal peak value of the lateral acceleration is decreased from $-2.9 \mathrm{~m} / \mathrm{s} 2$ to $-2.5 \mathrm{~m} / \mathrm{s} 2$ finally to $-1.1 \mathrm{~m} / \mathrm{s} 2$ respectively in the models of passive, two-system and three-system. The amplitude in the three-system model has been reduced by $50 \%$ than that in the passive system. The improvement of the lateral acceleration has greatly decreased the possibility of body sideslip and increased driving safety.

\section{Summary}

The simulation results based on the three-system model are best in the different chassis model of passive, two-system and three-system. The body sideslip angle, the yawing angular velocity and the lateral acceleration in the three-system model have all been decreased which has improved the vehicle handling stability and the driving safety. In future, the design for the three-system model should be considered some uncertainties in design process to improve the robustness of vehicle performance.

\section{Acknowledgements}

This work was financially supported by the Chinese National Natural Science Foundation (51005167) and Tianjin University of Technology and Education Science Foundation (KJY14-01).

\section{References}

[1] XIA Chang-gao, YANG Xi-he. Integrated Control System of 4WS and Active-suspension of Vehicles. Tractor \& Farm Transporter, 34 (2007) 63-69.

[2] Liu Li, Luo Yugong, Jiang Qingyun, etc. Integrated Chassis Control with AFS /DYC Based on Generalized Predictive Theory. Automotive Engineering, 33 (2011) 52-55.

[3] YANG Liu-qing, CHEN Wu-wei, CHU Chang-bao. Research on the conjoint control of the vehicle's active suspension and anti-lock braking system. Hefei University of TECHNOLOGY (Natural Science), 29 (2006) 767-771.

[4] Feng Jinzhi, Yu fan, Li Jun. An Investigation on Integrated Control of Vehicle Anti-lock Braking System and Active Suspension. Chinese Society of Agricultural Machinery, 33 (2002) 15-19.

[5] CHAI Mu, DONG En-guo, LI Zhen-xing. Research on fuzzy-PID control theory of vehicle active suspension. Machine Design, 30 (2012) 1-4.

[6] Gordon T J. An integrated strategy for the control of a full vehicle active suspension system. Vehicle System Dynamics, 25 (2010) 229-242. 